\title{
Effet de Différentes Doses d'Engrais Minéraux sur le Rendement de deux Variétés de Patate Douce [Ipomoea batatas (L) Lam] à Bouaké, Centre de la Côte d'Ivoire
}

\author{
Dr. Evrard Brice Konan Dibi, \\ Station de Recherche sur les Cultures Vivrières, Centre National de \\ Recherche Agronomique, Bouaké, Côte d'Ivoire \\ Jean Hugues Martial Kouassi, Msc \\ Unité de Formation et de Recherche en Agroforesterie, Laboratoire de \\ Physiologie Végétale, Université Jean Lorougnon Guédé de Daloa, \\ Daloa, Côte d'Ivoire \\ Dr. Emmanuel Kouadio N'Goran, \\ Station Coton, Centre National de Recherche Agronomique, \\ Bouaké, Côte d'Ivoire \\ Dr. Brice Sidoine Essis, \\ Dr. Boni N'zue, \\ Dr. Michel Amani Kouakou,
}

Station de Recherche sur les Cultures Vivrières, Centre National de

Recherche Agronomique, Bouaké, Côte d'Ivoire

Doi:10.19044/esj.2019.v15n33p135 URL:http://dx.doi.org/10.19044/esj.2019.v15n33p135

\section{Résumé}

L'étude a été conduite sur deux campagnes (2015/2016 et 2016/2017) afin d'évaluer l'effet de l'apport d'engrais minéraux (NPK 15-15-15 et 12-2222) sur le rendement de deux variétés de patate douce à Bouaké. L'essai a été conduit selon un dispositif expérimental split-plot comportant deux variétés de patate douce (Tib-440060 et Irene) et cinq niveaux de fertilisation $(0,200$, $300,400,500 \mathrm{~kg} / \mathrm{ha}$ ) en trois répétitions. Les résultats ont montré que les engrais minéraux ont eu un effet positif sur les composantes du rendement des racines tubéreuses. Les résultats de l'étude ont également montré que l'apport d'engrais minéraux n'a pas modifié les paramètres organoleptiques mesurés à savoir le goût, l'aspect, la cuisson et la contenance en eau de la patate douce. La variété Irene semble plus s'accommoder à la fertilisation minérale par rapport à la variété Tib-440060.

Mots clés: Ipomoea batatas, Engrais minéraux, Rendement, Qualité organoleptique, Bouaké 


\title{
Effect of Different Rates of Mineral Fertilizer on the Yield of Two Varieties of Sweet Potato [Ipomoea batatas (L) Lam] in Bouaké, Central Côte d'Ivoire
}

\author{
Dr. Evrard Brice Konan Dibi, \\ Station de Recherche sur les Cultures Vivrières, Centre National de \\ Recherche Agronomique, Bouaké, Côte d'Ivoire \\ Jean Hugues Martial Kouassi, Msc \\ Unité de Formation et de Recherche en Agroforesterie, Laboratoire de \\ Physiologie Végétale, Université Jean Lorougnon Guédé de Daloa, \\ Daloa, Côte d'Ivoire \\ Dr. Emmanuel Kouadio N'Goran, \\ Station Coton, Centre National de Recherche Agronomique, \\ Bouaké, Côte d'Ivoire \\ Dr. Brice Sidoine Essis, \\ Dr. Boni N'zue, \\ Dr. Michel Amani Kouakou, \\ Station de Recherche sur les Cultures Vivrières, Centre National de \\ Recherche Agronomique, Bouaké, Côte d'Ivoire
}

\begin{abstract}
The study was conducted over two seasons (2015/2016 and 2016/2017) to assess the effect of mineral fertilizer inputs (NPK 15-15-15 and 12-22-22) on the yield of two sweet potato varieties in Bouaké. The test was conducted using a split-plot experimental design with two varieties of sweet potato (Tib-440060 and Irene) and five levels of fertilization (0, 200, 300, 400, $400,500 \mathrm{~kg} / \mathrm{ha}$ ) in three repetitions. The results showed that mineral fertilizers had a positive effect on the yield components of tuberous roots. The results of the study also showed that the use of mineral fertilizers did not modify the organoleptic parameters measured, namely the taste, appearance, cooking and water content of the sweet potato. Irene variety seems to be more suited to mineral fertilization than the Tib-440060 variety.
\end{abstract}

Keywords: Ipomoea batatas, mineral fertilizers, yield, organoleptic quality, Bouaké 


\section{Introduction}

La patate douce (Ipomoea batatas) est une plante économiquement importante de la famille des Convolvulaceae composée d'environ 50 genres et 1000 espèces (Scott \& Ewell, 1992). C'est l'une des plantes à racines et tubercules les plus importantes en Afrique subsaharienne avec des utilisations domestiques et industrielles. Sa valeur nutritionnelle dépasse de loin l'igname, le manioc et le taro (Onwueme, 1978). En Afrique, la patate douce devient de plus en plus une culture économique importante en raison de son potentiel, de lutte contre la pauvreté, de réduction de la cécité (en utilisant des variétés à chair orange) et d'amélioration de l'état nutritionnel des populations rurales de manière peu coûteuse et durable (Brobbey, 2015).

En Côte d'Ivoire, la patate douce est très peu cultivée mais reste toutefois une culture de rente et un aliment important dans certaines régions (Dibi et al., 2016). Bien que la culture de la patate douce soit facile à cultiver, elle est confrontée à certaines contraintes de production. Parmi les nombreuses difficultés figure la baisse de la fertilité des sols due à la disparition des jachères de longue durée (Hinvi et al., 2000). En règle générale, la patate douce est une plante rustique qui tolère les terres pauvres ou marginales, mais l'amélioration de la fertilité du sol augmente sa croissance et sa productivité (Onunka et al., 2012). Bien que le fumier biologique pourrait être utile dans la production de la patate douce, son volume et la libération lente des éléments nutritifs sont difficile à gérer. Bien que l'engrais minéral soit relativement coûteux, sa libération rapide d'éléments nutritifs et sa manipulation facile en font une meilleure option, surtout pour la production commerciale. De plus, la patate douce est une culture de courte durée qui nécessite un engrais inorganique qui libère les éléments nutritifs dans le sol pour une utilisation plus rapide par les plantes. Par conséquent, l'utilisation d'engrais inorganiques serait très probablement plus efficace pour augmenter le rendement. Aussi la réaction des différentes variétés améliorées de patates douces aux engrais minéraux pourrait également varier. Les expérimentations en matière de fertilisation sur les variétés de patates douces récemment introduite par le CNRA, sont rares et peu documentés, ce qui nécessite des études dans ce sens. L'étude a donc été entreprise pour évaluer l'effet de différentes doses d'engrais inorganiques sur le rendement des variétés de patate douce à Bouaké.

\section{Matériel et Méthodes}

Cette étude a été conduite à la Station de Recherche sur les Cultures Vivrières (SRCV) du Centre National de Recherche Agronomique (CNRA) de Bouaké. Deux variétés de patates douces à savoir Irene et TIB-440060 ont été assignées à différents engrais minéraux à savoir le NPK 151515 + 28SO3 $+2 \mathrm{MgO}$ et le NPK $12222222+2 \mathrm{SO} 3+1 \mathrm{MgO}+5 \mathrm{CaO}$ à des doses de 200 ; 
300 ; 400 et $500 \mathrm{Kg} / \mathrm{ha}$ (Tableau 1). L'essai a été conduit pendant deux années consécutives (2016 et 2017) selon un dispositif en split-plot à trois répétitions. Dans ce dispositif, deux facteurs ont été étudiés. Un facteur principal constitué de deux variétés (Irène et TIB-440060) et un facteur secondaire constitué de fertilisants. Au total huit traitements ont été testés. Les blocs séparés par une allée de 2 m étaient composés de deux sous blocs séparés par des allées de 1,5 $\mathrm{m}$. Chaque sous bloc était composé de 9 parcelles élémentaires distants de 1 $\mathrm{m}$. Une parcelle élémentaire était constituée de seize (16) buttes reparties sur une surface de $9 \mathrm{~m} 2$ contenant 48 boutures en raison de trois boutures/butte. La taille des boutures variait de 15 à $30 \mathrm{~cm}$ portant 3 ou 4 nœuds. Les engrais ont été appliqués manuellement sur les monticules 21 jours après la plantation des boutures. La récolte des patates douces est intervenue 180 jours après plantation. Après la récolte, un test de dégustation a été réalisé pour apprécier l'aspect, la cuisson, la contenance en eau et le goût des racines récoltées selon un code de notation de 1 à 3 (Tableau 2). Les données ont été recueillies sur différents paramètres et ont fait l'objet d'analyse de variance (ANOVA) à deux facteurs à l'aide du logiciel Statistica version 7.1. En cas de différence significative, le test de Fisher au seuil de $5 \%$ a été utilisé pour classer les moyennes en groupes homogènes. Un test de Mann whitney a permis de comparer les moyennes deux à deux.

Tableau 1: Liste des différents traitements appliqués

\begin{tabular}{c|c|c}
\hline \multirow{2}{*}{ Traitements } & \multicolumn{2}{|c}{ Engrais minéraux } \\
\cline { 2 - 3 } & NPK 15 15 15 + & NPK 12 22 22 $+2 \mathrm{SO} 3+$ \\
$28 \mathrm{SO} 3+2 \mathrm{MgO}(\mathbf{k g} / \mathbf{h a})$ & $1 \mathrm{MgO}+5 \mathrm{CaO}(\mathbf{k g} / \mathbf{h a})$ \\
\hline Temoin & 0 & 0 \\
$\mathrm{~T}_{1}$ & 200 & 0 \\
$\mathrm{~T}_{2}$ & 300 & 0 \\
$\mathrm{~T}_{3}$ & 400 & 0 \\
$\mathrm{~T}_{4}$ & 500 & 0 \\
$\mathrm{~T}_{5}$ & 0 & 200 \\
$\mathrm{~T}_{6}$ & 0 & 300 \\
$\mathrm{~T}_{7}$ & 0 & 400 \\
$\mathrm{~T}_{8}$ & 0 & 500 \\
\hline
\end{tabular}

Tableau 2: Echelle de notation du test de dégustation

\begin{tabular}{|c|c|c|c|c|}
\hline \multirow{2}{*}{ Mets } & \multirow[t]{2}{*}{$\begin{array}{l}\text { Critères } \\
\text { d'appréciation }\end{array}$} & \multicolumn{3}{|c|}{$\begin{array}{c}\text { Correspondance des niveaux de } \\
\text { l'échelle } \\
\text { d'appréciation }\end{array}$} \\
\hline & & 1 & 2 & 3 \\
\hline \multirow{4}{*}{$\begin{array}{l}\text { Patate } \\
\text { bouillie }\end{array}$} & Aspect & Bon & Moyen & Pas bon \\
\hline & Cuisson & Bon & Moyen & Pas bon \\
\hline & Gout & Bon & Moyen & Pas bon \\
\hline & $\begin{array}{l}\text { Contenance en } \\
\text { eau }\end{array}$ & Bon & Moyen & Pas bon \\
\hline
\end{tabular}




\section{Résultats}

Effet de différentes doses d'engrais sur le nombre de racines commercialisables et non commercialisables de deux variétés de patate douce

Les nombres de racines commercialisables et non commercialisables sont restés statiquement identiques entre les différentes doses de fertilisants des deux engrais inorganiques (Tableau 3). Cependant au niveau des variétés, les résultats ont montré une différence significative $(\mathrm{p}=0,000)$ chez les racines commercialisables. La variété Tib-440060 a enregistré un nombre élevé de racines commercialisables avec en moyenne 263,5 par rapport à la variété Irene (192).

Tableau 3. Effet de différentes doses d'engrais sur le nombre de racines commercialisables et non commercialisables de deux variétés de patate douce

\begin{tabular}{cccccc}
\hline & & $\begin{array}{c}\text { Nombre de racines } \\
\text { commercialisables } \\
(>\mathbf{4} \mathbf{~ c m})\end{array}$ & $\begin{array}{c}\text { Nombre de racines } \\
\text { non }\end{array}$ \\
& & \multicolumn{2}{c}{$\begin{array}{c}\text { comercialisables } \\
(\leq \mathbf{4} \text { cm) }\end{array}$} \\
\cline { 2 - 6 } & Traitement & V1 & V2 & V1 & V2 \\
& S & & & & \\
\hline & Témoin & 263,5 & 139 & 124,5 & 82 \\
& T1 & 214,5 & 121,5 & 104,5 & 62,5 \\
& T2 & 245,5 & 150 & 114,5 & 74 \\
NPK 15-15-15 & T3 & 214,5 & 156 & 87 & 139 \\
& T4 & 249 & 126,5 & 135 & 68 \\
\hline & T5 & 231,5 & 171,5 & 121,5 & 106,5 \\
NPK 12-22-22 & T6 & 230 & 139,5 & 74,5 & 101,5 \\
& T7 & 254,5 & 176 & 123,5 & 75 \\
& T8 & 226 & 192 & 133 & 113 \\
\cline { 2 - 6 } & Moyenne & 236,55 & 152,44 & 113,11 & 91,27 \\
& CV $(\%)$ & 28,54 & 34,43 & 40,78 & 71,61 \\
\hline
\end{tabular}

*Les moyennes en colonnes affectées de la même lettre ne diffèrent pas au seuil de $5 \%$ (test de Fisher), Tib-440060 (V1), Irene (V2)

\section{Effet de différentes doses d'engrais sur le rendement de deux variétés de patate douce}

Les résultats de l'effet des engrais et des variétés sur le rendement sont consignés dans le tableau 4. Les résultats obtenus sont statisquement différents au niveau du nombre de racines par plant. Le nombre le plus élévé de racines par plant $(3,08)$ a été obtenu par la variété Tib-440060 avec le T7 $(400 \mathrm{~kg} / \mathrm{ha}$ NPK 12-22-22) tandis que le nombre le plus faible de racines $(2,43)$ a été obtenu avec le T6 (300 kg/ha NPK 12-22-22). Quant à la fertilisation à base de NPK 15-15-15, c'est le T4 (500 kg/ha) qui a enregistré le plus grand et le plus petit nombre avec respectivement les variétés Tib-440060 $(2,98)$ et Irene $(1,98)$. 
Dans le cas de la variété, le nombre le plus élévé de racines par plant $(2,78)$ a été obtenu avec le T8 (500 kg/ha NPK 12-22-22) par la variété Irene et le plus faible $(1,96)$ avec le T1 $(200 \mathrm{~kg} / \mathrm{ha}$ NPK 15-15-15).

Le rendement des différents racines fraîches n'a pas varié de façon significative d'un traitement à l'autre chez les deux variétés. Cependant les rendements ont varié au niveau de la variété. Dans le cas de la variété Tib440060 , le rendement maximal (15 t/ha) a été enregistré avec le T4 (500 kg/ha NPK 15-15-15) tandis que le plus faible (9,68 t/ha) a été obtenu avec le T6 (300 kg/ha NPK 12-22-22). Avec la variété Irene, le rendement maximal (19,34 t/ha) a été obtenu avec le T5 (200 kg/ha NPK 12-22-22) et le plus faible (9,68 t/ha) avec le T4 (500 kg/ha NPK 15-15-15).

Tableau 4: Effet de différentes doses d'engrais sur le rendement de deux variétés de patate douce

\begin{tabular}{|c|c|c|c|c|c|c|c|}
\hline & \multirow[b]{2}{*}{$\begin{array}{c}\text { Traitem } \\
\text { ents }\end{array}$} & \multicolumn{2}{|c|}{$\begin{array}{c}\text { Nombre de racines } \\
\text { par plant }\end{array}$} & \multicolumn{2}{|c|}{$\begin{array}{l}\text { Poids moyen par } \\
\text { parcelle }(\mathrm{kg})\end{array}$} & \multicolumn{2}{|c|}{$\begin{array}{c}\text { Rendement } \\
\text { (t/ha) }\end{array}$} \\
\hline & & V1 & $\mathrm{V} 2$ & V1 & V2 & V1 & V2 \\
\hline \multirow{5}{*}{$\begin{array}{c}\text { NPK 15-15- } \\
15\end{array}$} & Témoin & $2,91^{a b}$ & $\begin{array}{c}2,38 \\
\text { bcde }\end{array}$ & $16,75^{\mathrm{a}}$ & $19,75^{\mathrm{a}}$ & $10,46^{a}$ & $12,34^{\mathrm{a}}$ \\
\hline & $\mathrm{T} 1$ & $2,84 \mathrm{abc}$ & $1,96^{\mathrm{e}}$ & $16,75^{\mathrm{a}}$ & $18,55^{\mathrm{a}}$ & $10,46^{\mathrm{a}}$ & $11,59^{\mathrm{a}}$ \\
\hline & $\mathrm{T} 2$ & $2,81 \mathrm{abc}$ & $\begin{array}{c}2,31 \\
\text { bcde }\end{array}$ & $17,5^{\mathrm{a}}$ & $20,8^{a}$ & $10,93^{\mathrm{a}}$ & $13^{\mathrm{a}}$ \\
\hline & $\mathrm{T} 3$ & 2,67 abcd & $\begin{array}{l}2,63 \\
\text { abcde }\end{array}$ & $15,5^{\mathrm{a}}$ & $26,1^{a}$ & $9,68^{a}$ & $16,31^{\mathrm{a}}$ \\
\hline & $\mathrm{T} 4$ & $2,98^{\mathrm{ab}}$ & $1,98 \mathrm{de}$ & $24^{\mathrm{a}}$ & $15,5^{\mathrm{a}}$ & $15^{\mathrm{a}}$ & $9,68^{\mathrm{a}}$ \\
\hline \multirow{6}{*}{$\begin{array}{c}\text { NPK 12-22- } \\
22\end{array}$} & T5 & $2,73^{a b c}$ & $\begin{array}{c}2,47 \\
\text { abcde }\end{array}$ & $20^{a}$ & $30,95^{\mathrm{a}}$ & $12,50^{\mathrm{a}}$ & $19,34^{\mathrm{a}}$ \\
\hline & T6 & 2,43 abcde & $2,20 \mathrm{de}$ & $15,5^{\mathrm{a}}$ & $24,55^{\mathrm{a}}$ & $9,68^{\mathrm{a}}$ & $15,34^{\mathrm{a}}$ \\
\hline & $\mathrm{T} 7$ & $3,08^{\mathrm{a}}$ & $\begin{array}{l}2,47 \\
\text { abcde }\end{array}$ & $17,25^{\mathrm{a}}$ & $18,65^{\mathrm{a}}$ & $10,78^{a}$ & $11,65^{\mathrm{a}}$ \\
\hline & T8 & $2,83^{a b c}$ & $2,78 \mathrm{abc}$ & $19,50^{\mathrm{a}}$ & $24,25^{\mathrm{a}}$ & $12,18^{\mathrm{a}}$ & $15,15^{\mathrm{a}}$ \\
\hline & Moyenne & 2,80 & 2,35 & 18,08 & 22,12 & 11,29 & 13,82 \\
\hline & $\mathrm{CV}(\%)$ & 10,35 & 15,31 & 29,31 & 73,59 & 29,29 & 76,9 \\
\hline
\end{tabular}

*Les moyennes en colonnes affectées de la même lettre ne diffèrent pas au seuil de $5 \%$ (test de Fisher), Tib-440060 (V1), Irene (V2)

Effet de différentes doses d'engrais sur la matière sèche de deux variétés de patate douce

La teneur moyenne en matière sèche des racines fraîches a varié de $33,41 \%$ à 39,21\% pour tous les niveaux de fertilisations appliqués sur les deux variétés (Tableau 5). La teneur en matière sèche de la racine fraîche à 200 kg/ha NPK 15-15-15 (39,21 \%) obtenu avec la variété Tib-440060 était significativement plus élevée que celle de tous les autres traitements comme le montre le tableau 3. La teneur en matière sèche la plus faible $(33,41 \%)$ a été enrégistré à $300 \mathrm{~kg} / \mathrm{ha}$ NPK 12-22-22 avec la variété Tib-440060. Dans le cas de la variété Irene, la teneur en matière sèche a oscillé entre $36,18 \%$ et 
$33,74 \%$. La plus forte teneur $(36,18 \%)$ a été obtenu à $300 \mathrm{~kg} / \mathrm{ha}$ NPK $12-22-$ 22 alors que la plus faible $(33,74 \%)$ a été enregistré avec $200 \mathrm{~kg} / \mathrm{ha}$ NPK 15 $15-15$.

Tableau 5: Effet de différentes doses d'engrais sur la matière sèche de deux variétés de patate douce

\begin{tabular}{cccc}
\hline & & \multicolumn{2}{c}{ Taux de matière sèche (\%) } \\
\cline { 2 - 4 } NPK 15-15- & Traitements & V1 & V2 \\
\hline 15 & Témoin & $35,47^{\mathrm{ab}}$ & $35,33^{\mathrm{ab}}$ \\
& $\mathrm{T} 1$ & $39,21^{\mathrm{a}}$ & $33,74^{\mathrm{b}}$ \\
& $\mathrm{T} 2$ & $35,33^{\mathrm{ab}}$ & $35,42^{\mathrm{ab}}$ \\
& $\mathrm{T} 3$ & $35,76^{\mathrm{ab}}$ & $36,63^{\mathrm{ab}}$ \\
& $\mathrm{T} 4$ & $34,76^{\mathrm{ab}}$ & $35,66^{\mathrm{ab}}$ \\
\hline NPK 12-22- & $\mathrm{T} 5$ & $33,60^{\mathrm{b}}$ & $34,48^{\mathrm{ab}}$ \\
$\mathbf{2 2}$ & & & \\
& $\mathrm{T} 6$ & $33,41^{\mathrm{b}}$ & $36,18^{\mathrm{ab}}$ \\
& $\mathrm{T} 7$ & $34,48^{\mathrm{ab}}$ & $36,08^{\mathrm{ab}}$ \\
& $\mathrm{T} 8$ & $35,84^{\mathrm{ab}}$ & $35,12^{\mathrm{ab}}$ \\
\cline { 2 - 4 } & Moyenne & 35,31 & 35,40 \\
& CV $(\%)$ & $7,41^{\mathrm{a}}$ & 5,28 \\
\hline
\end{tabular}

* Les moyennes en colonnes affectées de la même lettre ne diffèrent pas au seuil de $5 \%$ (test de Fisher), Tib-440060 (V1), Irene (V2)

\section{Efficacité des engrais minéraux}

Les résultats de l'analyse de la figure 1 ont montré que le T5 $(200 \mathrm{~kg} / \mathrm{ha}$ NPK 12-22-22) qui a enregistré le meilleur rendement avec la variété Irene a été le traitement le plus efficace avec un coefficient d'efficacité de 13. Il est suivi du traitement T3 (400 kg/ha NPK 15-15-15) avec un coefficient d'efficacité de 8 . Le coefficient d'éfficacité des autres traitements a varié entre 1 et 3 .

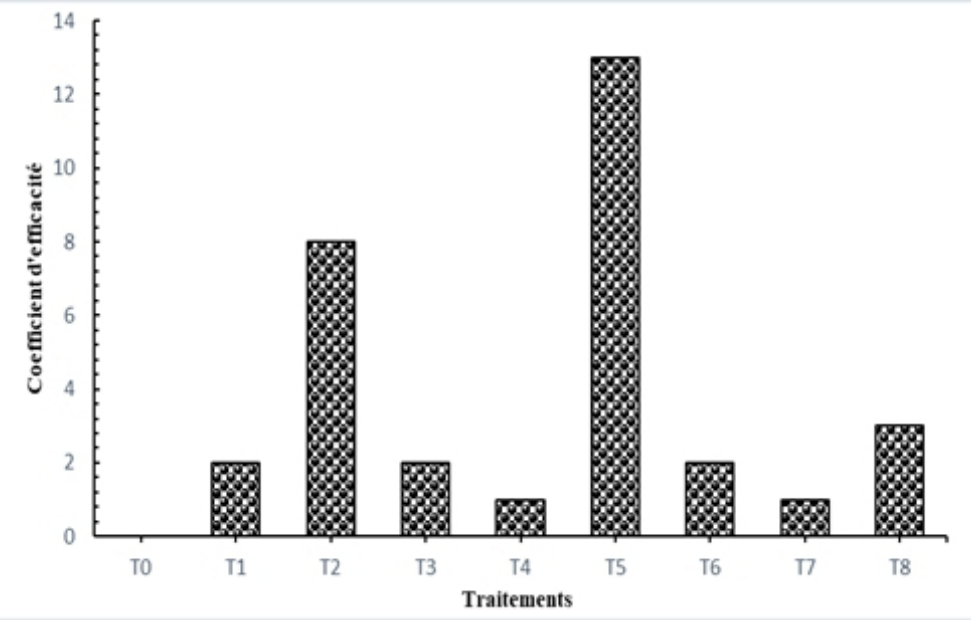

Figure 1: Evolution du coefficient d'efficacité en fonction des traitements 


\section{Effet de différentes doses d'engrais sur la qualité organoleptique de deux variétés de patate douce}

Le tableau 6 présente les résultats obtenus à l'issu des tests organoleptiques. Les engrais n'ont pas affecté la qualité organoleptique de la patate douce $(\mathrm{p}>0,05)$. Par contre, concernant la variété, les résultats obtenus ont montré un effet significatif au niveau de la contenance en eau et du goût de la patate bouillie $(\mathrm{p}<0,05)$. En effet, la variété Irene a présenté à $200 \mathrm{~kg} / \mathrm{ha}$ NPK 12-22-22 des mets ayant un bon goût $(1,11)$ avec une contenance en eau moyenne $(1,47)$ tandis qu'au niveau de la cuisson et de l'aspect, aucune différence significative n'a été observée $(p>0,05)$. Au niveau de l'engrais de formulation NPK 15-15-15, c'est la variété Irene qui a presenté un mets avec un bon goût $(1,19)$ et une contenance en eau moyenne $(1,33)$ à la dose de 500 $\mathrm{kg} / \mathrm{ha}$.

Tableau 6: Effet de différentes doses d'engrais sur la qualité organoleptique de deux variétés de patate douce

\begin{tabular}{cccccc}
\hline & \multicolumn{3}{c}{ Aspect } & \multicolumn{2}{c}{ Goût } \\
\cline { 2 - 6 } NPK 15-15-15 & Traitements & V1 & V2 & V1 & V2 \\
\hline & Témoin & 1,83 & 1,33 & $2,04^{\mathrm{d}}$ & $1,38^{\mathrm{abcd}}$ \\
& T1 & 1,61 & 1,75 & $1,83^{\mathrm{bcd}}$ & $1,41^{\mathrm{abcd}}$ \\
& T2 & 1,77 & 1,75 & $1,90^{\mathrm{cd}}$ & $1,47^{\mathrm{abcd}}$ \\
& T3 & 1,89 & 1,72 & $1,66^{\mathrm{abcd}}$ & $1,55^{\mathrm{abcd}}$ \\
& T4 & 1,49 & 1,41 & $1,97^{\mathrm{d}}$ & $1,19^{\mathrm{ab}}$ \\
\hline NPK 12-22-22 & T5 & 1,88 & 1,30 & $1,81^{\mathrm{bcd}}$ & $1,11^{\mathrm{a}}$ \\
& T6 & 1,60 & 1,61 & $1,75^{\mathrm{abcd}}$ & $1,27^{\mathrm{abc}}$ \\
& T7 & 1,83 & 1,60 & $1,91^{\mathrm{cd}}$ & $1,60^{\mathrm{abcd}}$ \\
& T8 & 1,60 & 1,55 & $1,79^{\mathrm{bcd}}$ & $1,22^{\mathrm{ab}}$ \\
\cline { 2 - 6 } & Moyenne & 1,72 & 1,55 & 1,85 & 1,35 \\
& CV $(\%)$ & 15,69 & 19,87 & 17,83 & 14,07
\end{tabular}

*Les moyennes en colonnes affectées de la même lettre ne diffèrent pas au seuil de $5 \%$ (test de Fisher), Tib-440060 (V1), Irene (V2) 
Tableau 7: Effet de différentes doses d'engrais sur la qualité organoleptique de deux variétés de patate douce

\begin{tabular}{cccccc}
\hline & \multicolumn{2}{c}{ Cuisson } & \multicolumn{2}{c}{ Teneur en eau } \\
\cline { 2 - 6 } & Traitements & V1 & V2 & V1 & V2 \\
\hline \multirow{3}{*}{ NPK 15-15-15 } & Témoin & 1,44 & $1,50^{\mathrm{a}}$ & $1,55^{\mathrm{abc}}$ & $1,49^{\mathrm{abc}}$ \\
& T1 & 1,38 & $1,36^{\mathrm{a}}$ & $1,36^{\mathrm{ab}}$ & $1,52^{\mathrm{abc}}$ \\
& T2 & 1,38 & $1,49^{\mathrm{a}}$ & $1,49^{\mathrm{abc}}$ & $1,60^{\mathrm{abc}}$ \\
& T3 & 1,22 & $1,71^{\mathrm{a}}$ & $1,16^{\mathrm{a}}$ & $1,88^{\mathrm{c}}$ \\
& T4 & 1,44 & $1,30^{\mathrm{a}}$ & $1,60^{\mathrm{abc}}$ & $1,33^{\mathrm{ab}}$ \\
\hline NPK 12-22-22 & T5 & 1,71 & $1,46^{\mathrm{a}}$ & $1,66^{\mathrm{bc}}$ & $1,47^{\mathrm{abc}}$ \\
& T6 & 1,49 & $1,33^{\mathrm{a}}$ & $1,27^{\mathrm{ab}}$ & $1,61^{\mathrm{abc}}$ \\
& T7 & 1,60 & $1,33^{\mathrm{a}}$ & $1,66^{\mathrm{bc}}$ & $1,44^{\mathrm{abc}}$ \\
& T8 & 1,49 & $1,61^{\mathrm{a}}$ & $1,55^{\mathrm{abc}}$ & $1,27^{\mathrm{ab}}$ \\
\cline { 2 - 6 } & Moyenne & 1,46 & $1,45^{2}$ & 1,47 & 1,51 \\
& CV $(\%)$ & 16,43 & 15,17 & 16,21 & 15,23
\end{tabular}

* Les moyennes en colonnes affectées de la même lettre ne diffèrent pas au seuil de $5 \%$ (test de Fisher), Tib-440060 (V1), Irene (V2)

\section{Discussion}

L'application des engrais minéraux a permis d'avoir une augmentation du rendement en racines tubéreuses fraîches par rapport au témoin sans engrais. Ce résultat est vraisemblablement dû à l'accessibilité directe des éléments nutritifs $\mathrm{N}$, $\mathrm{P}$ et $\mathrm{K}$ apportés au sol par l'engrais composé. Il est donc probable que les augmentations entre le témoin (T0) et les traitements T3 et T5 soient dues aux effets conjugués des trois éléments N, P et $\mathrm{K}$. Des résultats similaires ont été obtenus en Côte d'Ivoire sur le manioc (Bakayoko et al., 2009) et dans d'autres pays (Borah et al., 2010; Zoundi, 2012). Ces résultats ont montré que les rendements les plus élevés avaient été enregistrés par les apports d'engrais ayant des apports de N, P et K. De même Lompo \& Belem (2012) ont obtenu au Burkina Faso, les meilleurs rendements et les poids frais de racines tubéreuses avec la formule minérale de N60P30K100 sur la patate douce.

Par ailleurs, Loué (1977) a signalé que le potassium contribue à l'amélioration des calibres, tandis qu'une déficience en $\mathrm{K}$ entraîne une plus grande proportion de petits calibres. Plusieurs travaux, notamment ceux de Njoku et al. (2001) et Kebdani et al. (2014) ont également confirmé que l'apport de $\mathrm{N}$ et de $\mathrm{K}$ sont essentiels à la production de patate douce. De plus, le rendement des racines tubéreuses de la patate douce pourrait dépendre de la capacité photosynthétique du couvert foliaire. Cette capacité qu'à la plante à transloquer les assimilés de la feuille à la racine et de leur capacité à les assimiler. Il est donc possible que l'assimilation de la photosynthèse transloquée aux racines ait pu contribuer aux rendements élevés des racines du traitement T5 (Brobbey, 2015). 
Les résultats du test organoleptique ont montré qu'il n'y a pas eu d'effet des fertilisants sur la qualité organoleptique de la patate douce après fertilisation. Les conclusions tirées dans cette étude, vont dans le même ordre d'idée que celles rapportées par Soro et al. (2003), Aziadekey et al. (2014) qui ont montré que l'application de la fertilisation minérale a produit les meilleurs rendements en tubercules chez des variétés d'ignames, sans altérer leurs qualités organoleptiques.

La variation de la teneur de matière sèche chez les variétes (Irene 36,63 $\%$ et Tib-440060 39,21\%) et non chez les traitements indique que cette importante teneur en matière sèche chez ces variétés serait une caractéristique variétale propre à elles. Des résultats identiques ont été rapporté par Feruzi et al. (2001) chez des variétés cultivées à l'Est du Congo.

Les rendements augmentent avec l'apport d'engrais compris entre 200 et $400 \mathrm{~kg} / \mathrm{ha}$, mais au-delà les rendements baissent. Cette situation s'explique par le fait que la quantité d'éléments fertilisants apportées par l'engrais minéral va au-delà des besoins de la culture, et il se produit par conséquent une baisse de rendement liée à l'antagonisme entre les éléments nutritifs (Gros, 1967).

Autrement dit il y a une limite à l'emploi des fertilisants et d'une manière générale, à tous les facteurs de croissance. Ainsi, les doses de 200 $\mathrm{kg} / \mathrm{ha}$ de NPK 12-22-22 et $400 \mathrm{~kg} / \mathrm{ha}$ de NPK 151515 ont enregistré les meilleurs rendements par rapport aux doses de $500 \mathrm{~kg} / \mathrm{ha}$ NPK. En effet, pour tout fertilisant apporté, le coefficient d'efficacité des engrais aux différents traitements diminue avec les doses croissantes (Kimuni et al., 2014). Ces résultats montrent l'intérêt et la nécessité d'utiliser des doses faibles parce que les fortes doses ne donnent pas de bons rendements.

\section{Conclusion}

La fertilisation est nécessaire pour accroître la production de la patate douce. L'augmentation de cette production apparaît toutefois liée au type et à la dose d'engrais. Des résultats obtenus, il ressort que la fertilisation minérale améliore le rendement de la patate douce et ne modifie pas les qualités organoleptiques de celle-ci. Mais cette amélioration est liée à la variété. Ainsi la fertilisation minérale de la patate douce avec $200 \mathrm{~kg} / \mathrm{ha}$ (NPK 12-22-22) et $400 \mathrm{~kg} / \mathrm{ha}$ (NPK 15-15-15) ont permis d'obtenir un gain de rendement de racines tubéreuses élevé et la variété Irene semble bien s'accommoder à la fertilisation minérale. En référence aux rendements obtenus et au coefficient d'efficacité, les doses de $200 \mathrm{~kg} / \mathrm{ha}$ NPK 12-22-22 et $400 \mathrm{~kg} / \mathrm{ha}$ de NPK $15-$ 15-15 sont recommandé pour la production de patate douce. De plus la qualité organoleptique n'ayant pas été influencé par la fertilisation pourrait être une raison supplémentaire de motivation du paysan. Etant donné que les résultats obtenus dans cette étude sont pour un seul site, il serait judicieux d'évaluer de 
façon multi-locale l'effet de différentes combinaisons d'éléments fertilisants sur le rendement et la qualité des racines de patate douce.

\section{References :}

1. Aziadekey, M., Sogbedji, M., Odah, K., Amouzouvi, K. et Afanahin, K. (2014). Effets de la fumure organo-minérale sur la production et les qualités organoleptiques de deux variétés d'igname du complexe dioscorea cayenensis-rotundata dans la région de kara au togo. European Scientific Journal edition vol.10, No.15: 178-191.

2. Bakayoko, S., Tschannen, A., Nindjin, C., Dao, D., Girardin, O. et Assa, A. (2009). Impact of water stress on fresh tuber yield and dry matter content of cassava (Manihot esculenta Crantz) in Côte d'Ivoire. African Journal of Agricultural Research 4: 021-027.

3. Borah, K. K., Bhuyan, B. et Sarma, H. (2010). Assessment of Soil Fertility Status in and Around the Tea Gardens of Undivided Darrang District, Assam. International Journal of Applied Environmental Sciences, $5 \mathrm{p}$.

4. Brobbrey, A. (2015). Growth, yield and quality factors of sweetpotato (Ipomoea batatas (1) lam) as affected by seedbed type and fertilizer applicationeting and utilization of root and tuber crops in africa. Master of philosophy in agronomy department of crop and soil sciences Kwame Nkrumah University of Science and Technology (Kumasi), 95p.

5. Dibi, K. E. B., Essis, B., N'Zué, B., Kouakou, A. M. et Zohouri, G. P. (2016). Appui à la promotion de la patate douce à chair orange en Côte d'Ivoire. Rapport final avenant du Projet / Agriculture familiale pour la nutrition et l'équité de genre (CHANGE). CNRA Bouaké. Janvier 2016, 16p.

6. Feruzi, M., Phemba, P., Ngongo, M., Hagenimana, V. et Lutaladio N. B. (2001). Evaluation post-récolte de six Génotypes de patate douce sélectionnés à l'Est du Congo. Afr. Crop.Sci. Journal. 9(1) : 33-39.

7. Gros, A. 1967. Engrais, guide pratique de la fertilisation, 4ème édition, Maison Rustique, Paris, France, 239p.

8. Hinvi, J. C. et Nonfon, R. (2000). La production et la commercialisation des semenceaux d'igname au Bénin : une nécessité de plus en plus incontournable. In l'igname et la pomme de terre en Afrique de l'Ouest. Actes d'un atelier sous régional sur l'igname et la pomme de terre, pp. 81-89.

9. Kebdani, B. et Missat, L. (2014). Etude de l'influence de type de la fertilisation et l'apport de fumure sur la culture de pomme de terre (Solanum tuberosum L) Mémoire de Master II En Agronomie Option 
production et amélioration végétales Université Abou Bekr Belkaid (Tlemcen), 83p.

10. Kimuni, L. N., Mwali, M. K., Mulembo, T. M., Lwalaba, J. W. L., Kanyenga, L. A., Katombe, J.L., ... Longanza, B. L. (2014). Effets de doses croissantes des composts de fumiers de poules sur le rendement de chou de Chine installé sur un sol acide de Lubumbashi. Journal of Applied Biosciences 77 : 6509-6522.

11. Loue, A. (1977). Fertilisation et nutrition minérale de la pomme de terre Mulhouse : S.C.P.A (direction technique), 147p.

12. Lompo, F. et Belem, J. (2012). Promotion of orange-fleshed sweetpotato to control vitamin A and antioxidant deficiencies in Burkina Faso. Annual report Year 3 Oct. 11-Sept. 12, 67p.

13. Njoku, J. C., Okpara, D. A. et Asiegbu, J. E. (2001). Growth and yield response of sweet potato to inorganic nitrogen and potassium in a tropical ultisol. Nigerian Agricultual Journal, 32: 30-41.

14. Onunka, N. A., Chukwu, L. I., Mbanasor, E. O. et Ebeniro, C. N. (2012). Effect of organic and inorganic manures and time of application on soil properties and yield of sweet potato in a tropical ultisol. Journal of Agriculture \& Social Reseach, 12(1), 182-193.

15. Onwueme, I. C. (1978). The tropical tuber crops. Yam, Cassava, Sweet potato and Cocoyams. Chichester, Royaume-Uni, Wiley, 234p.

16. Scott, G. J. et Ewell, P. E. (1992). Sweet potato in African food systems. In: Scott G., Ferguson P.I, and Herrera J.E (eds). Product Development for Root and Tuber Crops. Vol. III Africa. Proceedings on the workshop on Processing, Marketing, and Utilization of roots and Tuber Crops in Africa, held October 26 November 2, 1991 at the IITA, Ibadan, Nigeria, International Potato Center, Lima, Peru 170p.

17. Soro, D., Dao, D., Carsky, R., Asiedu, R., Tra, T. B., Assa, A. et Girardin, O. (2003). Amélioration de la production de l'igname à travers la fertilisation minérale en zone de savane de Côte d'Ivoire. Agronomie Africaine (numéro spécial, Atelier National sur l'igname d'octobre 2001) $4: 39-46$.

18. Zoundi, S. J. (2012). Agriculture vivrière : Les Africains confrontés à des choix controversés de modèles agricoles. Cahiers Agricultures 21:366-373. 